\title{
FORWARD DISPERSION RELATIONS FOR $\pi K$ SCATTERING AND THE $K_{0}^{*}(800)$ RESONANCE*
}

\author{
A. RODAS \\ Departamento de Física Teórica II, Universidad Complutense de Madrid \\ 28040 Madrid, Spain
}

(Received July 19, 2016)

We review the recent analysis of $\pi K$ scattering data in terms of forward dispersion relations, and also present the parameters of the strange resonances. This work consists of fits to the data that are constrained to satisfy analyticity requirements. The method yields a set of simple and consistent parameterizations that are compatible with forward dispersion relations up to $1.6 \mathrm{GeV}$ while still describing the data. We also obtain the pole parameters of the $K_{0}^{*}(800)$ and the $K^{*}(892)$ resonances.

DOI:10.5506/APhysPolBSupp.9.459

\section{Introduction}

A precise knowledge of $\pi K$ scattering is of interest since it is one of the main reactions involved in the final state of hadronic processes with net strangeness. It is also interesting by itself because it provides a test of Chiral Perturbation Theory [1] and other unitarized approaches [2]. Moreover, there is a renewed interest in $\pi K$ scattering from lattice QCD, where the main features, like threshold parameters [3], scattering phases and resonances [4], have been already calculated. Finally, our study of the scalar channel leads to a clear pole for the still controversial $K_{0}^{*}(800), \kappa$, which according to the PDG, needs confirmation.

From the experimental point of view, these processes cannot be directly measured and hence the data is plagued by systematic uncertainties. The data available in the bibliography [5] are clearly incompatible, furthermore, there are no data close to the threshold.

Our goal is to perform an analysis of this scattering process using only analytic constraints and data. The dispersive integral formalism is modelindependent and relates the value of the amplitude with an integral over

* Presented at "Excited QCD 2016", Costa da Caparica, Lisbon, Portugal, March 6-12, 2016. 
the whole real axis, increasing the precision and giving information even in regions where data are lacking or have large uncertainties. In addition, it relates different channels among themselves. Moreover, it is also useful to constrain threshold and resonance parameters.

Here, we review the recent work in collaboration with Peláez [6], which is based on Forward Dispersion Relations (FDR). As they are calculated at $t=0$, we can use this set of equations up to arbitrary energies in the real axis, providing a set of simple but powerful constraints for the fits. We consider two independent amplitudes, one symmetric and one antisymmetric under the $s \leftrightarrow u$ exchange that cover the isospin basis $T^{+}(s)=\left(T^{1 / 2}(s)+\right.$ $\left.2 T^{3 / 2}(s)\right) / 3=T^{I_{t}=0}(s) / \sqrt{6}$ and $T^{-}(s)=\left(T^{1 / 2}(s)-T^{3 / 2}(s)\right) / 3=T^{I_{t}=1}(s) / 2$. The symmetric amplitude has one subtraction and can be written as

$$
\begin{aligned}
& \operatorname{Re} T^{+}(s)=T^{+}\left(s_{\mathrm{th}}\right)+\frac{\left(s-s_{\mathrm{th}}\right)}{\pi} \\
& \times P \int_{s_{\mathrm{th}}}^{\infty} \mathrm{d} s^{\prime}\left[\frac{\operatorname{Im} T^{+}\left(s^{\prime}\right)}{\left(s^{\prime}-s\right)\left(s^{\prime}-s_{\mathrm{th}}\right)}-\frac{\operatorname{Im} T^{+}\left(s^{\prime}\right)}{\left(s^{\prime}+s-2 \Sigma_{\pi K}\right)\left(s^{\prime}+s_{\mathrm{th}}-2 \Sigma_{\pi K}\right)}\right],
\end{aligned}
$$

where $s_{\mathrm{th}}=\left(m_{\pi}+m_{K}\right)^{2}$. In contrast, the antisymmetric one does not require subtractions

$$
\operatorname{Re} T^{-}(s)=\frac{\left(2 s-2 \Sigma_{\pi K}\right)}{\pi} P \int_{s_{\mathrm{th}}}^{\infty} \mathrm{d} s^{\prime} \frac{\operatorname{Im} T^{-}\left(s^{\prime}\right)}{\left(s^{\prime}-s\right)\left(s^{\prime}+s-2 \Sigma_{\pi K}\right)} .
$$

We also include in our analysis 3 sum rules for threshold parameters (scattering lengths and slopes) in order to obtain the best possible result in this region, where there are no data. These integral equations allow us to obtain a set of precise scattering lengths. There can be observed [6] how this analysis leads to a set of threshold parameters compatible with the experimental measurements of the DIRAC Collaboration [7].

There exists previous dispersive analysis [8] that obtains the phase shifts of the scalar and vectorial channels by using Roy-Steiner equations (RS). However, these equations can only be applied in the low-energy region $(\sqrt{s} \leq$ $0.935 \mathrm{GeV})$.

\section{Method and results}

The approach used in this work follows the same steps as previous works done by our group for $\pi-\pi$ scattering [9].

(1) We first obtain simple fits for each partial wave independently, called Unconstrained Fits to Data (UFD), without including any model description. 
(2) We check the fulfillment of the dispersion relations to observe if there are some inconsistent data points that do not satisfy it.

(3) We finally impose these integral equations to obtain the final Constrained Fits to Data (CFD), where all the partial waves are related through the FDR and the sum rules.

In order to impose the FDRs, we define $d_{i}$ - the difference between the input and the output of each dispersion relation at the energy point $s_{i}$, whose uncertainties are $\Delta d_{i}$. We thus define the average discrepancies

$$
d_{T \pm}^{2}=\frac{1}{N} \sum_{i=1}^{N}\left(\frac{d_{i}}{\Delta d_{i}}\right)_{T^{ \pm}}^{2} .
$$

We introduce a penalty function that measures the difference between the UFD parameters and the CFD ones to describe also the data, obtaining the final $\chi^{2}$ function

$$
\chi^{2}=W^{2}\left(d_{T^{+}}^{2}+d_{T^{-}}^{2}\right)+d_{\mathrm{SR}}+\sum_{k}\left(\frac{p_{k}^{\mathrm{UFD}}-p_{k}}{\delta p_{k}^{\mathrm{UFD}}}\right)^{2} .
$$

The weight $W^{2}=12$ stands for the FDR that is just the number of degrees of freedom needed to describe the amplitude in the region of interest.

Figure 1 shows the total amplitudes and the huge improvement between the UFD and the CFD parametrizations; in Fig. 2, we show the difference between the fits to the data and the final results.

We show in Table I the average discrepancies for different energy regions. As it can be observed, the CFD result is very consistent below $1.6 \mathrm{GeV}$.

TABLE I

Fulfillment of Forward Dispersion Relations.

\begin{tabular}{l|cc|cc}
\hline \hline & \multicolumn{2}{|c|}{ UFD } & \multicolumn{2}{c}{ CFD } \\
& $d_{T^{+}}^{2}$ & $d_{T^{-}}^{2}$ & $d_{T^{+}}^{2}$ & $d_{T^{-}}^{2}$ \\
\hline$\sqrt{s_{\min }} \leq \sqrt{s} \leq m_{K}+m_{\eta}$ & 3.35 & 0.97 & 0.39 & 0.13 \\
$m_{K}+m_{\eta} \leq \sqrt{s} \leq 1.6 \mathrm{GeV}$ & 1.3 & 6.8 & 0.17 & 0.70 \\
$1.6 \mathrm{GeV} \leq \sqrt{s} \leq 1.74 \mathrm{GeV}$ & 14.6 & 12.8 & 8.0 & 0.5 \\
\hline$\sqrt{s_{\min }} \leq \sqrt{s} \leq 1.74 \mathrm{GeV}$ & 3.9 & 5.1 & 1.3 & 0.44
\end{tabular}



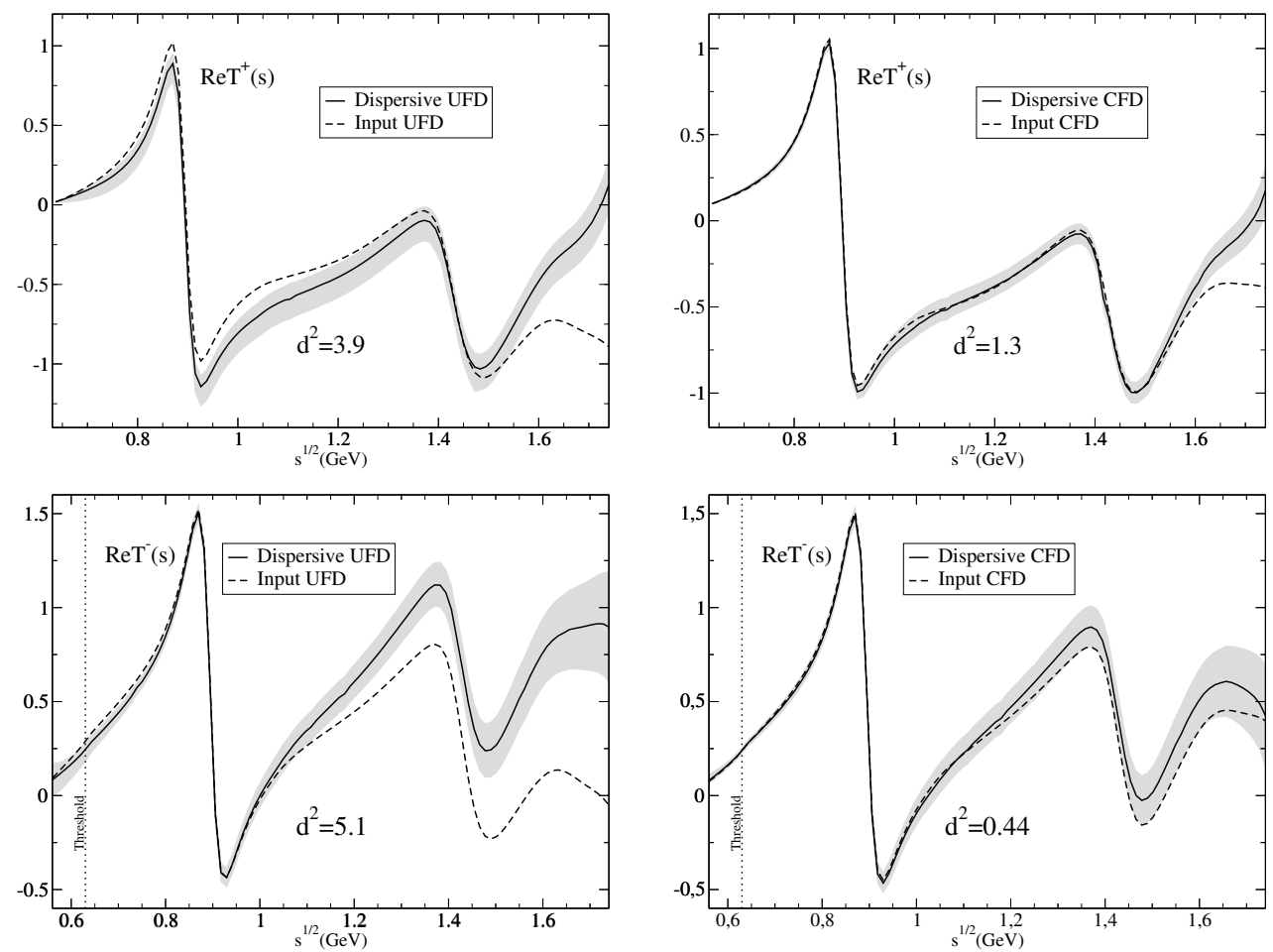

Fig. 1. Comparison between the input (fits) and the output (FDRs) for the total amplitudes $T^{+}$(top) and $T^{-}$(bottom). The gray bands describe the uncertainty of the difference between the input and the output.

Now, we use the CFD parameters to give the result for the most important threshold parameters, obtaining $m_{\pi} a_{0}^{1 / 2}=0.22 \pm 0.01$ and $m_{\pi} a_{0}^{3 / 2}=$ $-0.054_{-0.014}^{+0.010}$. We show here our results compared with the recent result measured by the Dirac Collaboration

$$
\begin{aligned}
& \frac{1}{3}\left(a_{0}^{1 / 2}-a_{0}^{3 / 2}\right)=0.11_{-0.04}^{+0.09} m_{\pi}^{-1} \quad(\text { DIRAC }), \\
& \frac{1}{3}\left(a_{0}^{1 / 2}-a_{0}^{3 / 2}\right)=0.091_{-0.005}^{+0.006} m_{\pi}^{-1} \quad(\mathrm{CFD}) .
\end{aligned}
$$

Using our conformal parametrization, we can also continue the partial waves to the complex plane calculating the position of the resonances in the second Riemann sheet. In Tables II and III, we show the mass, width and coupling of each elastic resonance, defined as $s_{\mathrm{R}}=\left(M_{\mathrm{R}}-i \Gamma_{\mathrm{R}} / 2\right)^{2}$ and $|g|^{2}=\left|16 \pi(2 l+1) \operatorname{Res}\left(t_{l}\left(s_{\mathrm{R}}\right)\right) /\left(2 q\left(s_{\mathrm{R}}\right)\right)^{2 l}\right|$. 

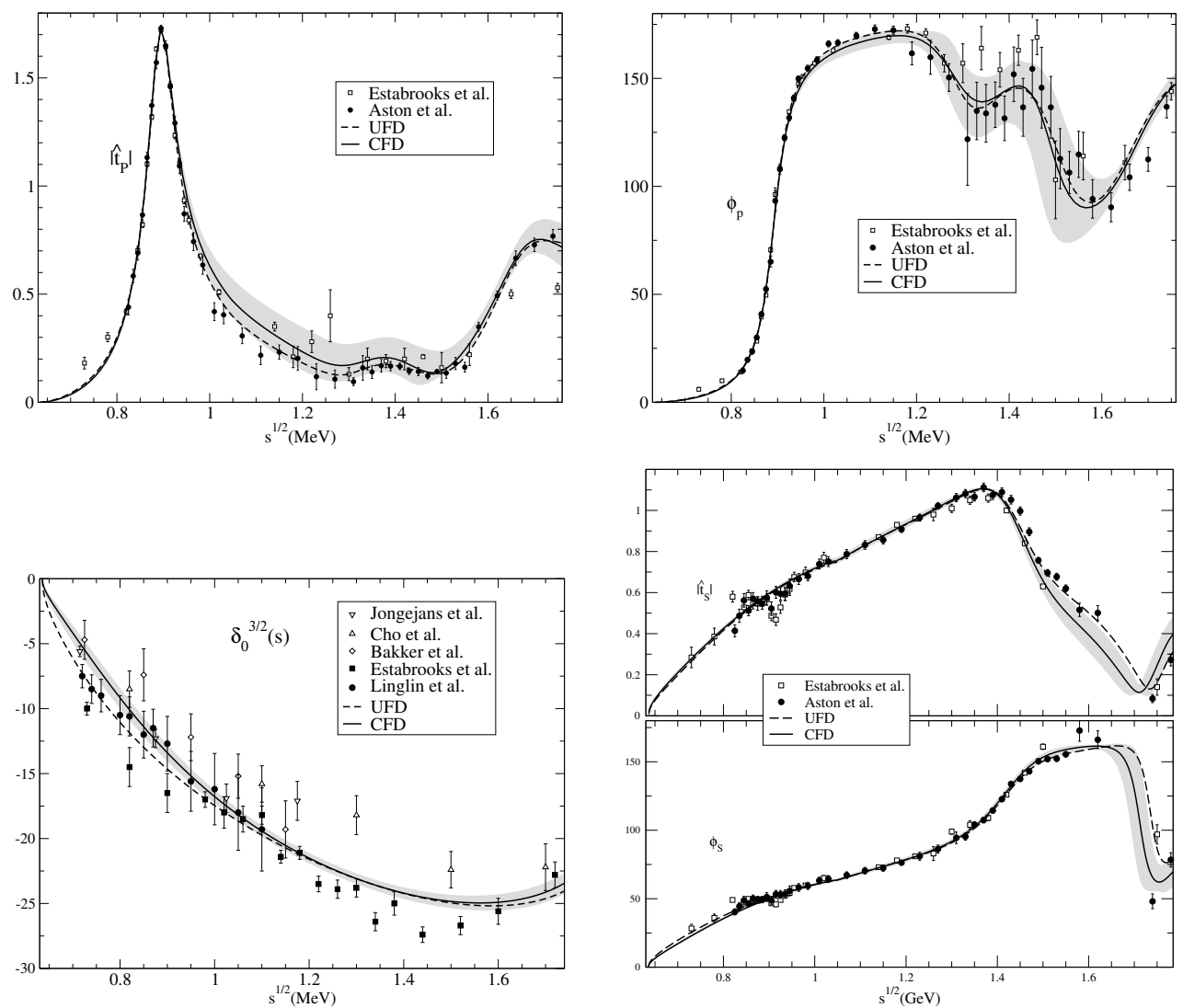

Fig. 2. Comparison between UFD and CFD fits for different partial waves, where $|\hat{t}|$ stands for the modulus, $\delta$ for the phase shift and $\phi$ for the total phase of each partial wave. The gray bands cover the errors of the parameters for each fit.

TABLE II

$K_{0}^{*}(800)$ pole parameters from the analytic continuation of the elastic parameterization.

\begin{tabular}{cccc}
\hline \hline Poles & Mass $[\mathrm{MeV}]$ & Width $[\mathrm{MeV}]$ & $|g|[\mathrm{GeV}]$ \\
\hline UFD & $673 \pm 15$ & $674 \pm 15$ & $5.01 \pm 0.07$ \\
CFD & $680 \pm 15$ & $668 \pm 15$ & $4.99 \pm 0.08$
\end{tabular}


$K^{*}(892)$ pole parameters from the analytic continuation of the elastic parameterization only.

\begin{tabular}{cccc}
\hline \hline Poles & Mass $[\mathrm{MeV}]$ & Width $[\mathrm{MeV}]$ & $|g|[\mathrm{GeV}]$ \\
\hline UFD & $893 \pm 1$ & $56 \pm 2$ & $5.95 \pm 0.07$ \\
CFD & $892 \pm 1$ & $58 \pm 2$ & $6.02 \pm 0.06$
\end{tabular}

\section{Outlook}

Figure 1 shows that the CFD set satisfies really well the dispersion relations up to $1.6 \mathrm{GeV}$. Above that energy, the differences between the input and the output require larger deviations from data as it is shown in Fig. 2.

Finally, the use of conformal mappings in the elastic region allows us to continue the partial waves to the second Riemann sheet. The values obtained for the parameters of the resonances are in agreement with other works listed in the PDG, although we obtain smaller uncertainties due to the small error of the CFD parameters.

A.R. is supported by the Spanish Projects No. FPA2011-27853-C02-02 and No. FPA2014-53375C2-2, and Red de Excelencia de Física Hadrónica FIS2014-57026-REDT.

\section{REFERENCES}

[1] J. Gasser, H. Leutwyler, Nucl. Phys. B 250, 465 (1985); V. Bernard, N. Kaiser, U.-G. Meißner, Nucl. Phys. B 357, 129 (1991).

[2] A. Dobado, J.R. Peláez, Phys. Rev. D 47, 4883 (1993); A. Gómez Nicola, J.R. Peláez, Phys. Rev. D 65, 054009 (2002); J.R. Peláez, Mod. Phys. Lett. A 19, 2879 (2004); J.A. Oller, E. Oset, Nucl. Phys. A 620, 438 (1997) [Erratum ibid. 652, 407 (1999)]; Phys. Rev. D 60, 074023 (1999); J.A. Oller, E. Oset, J.R. Peláez, Phys. Rev. Lett. 80, 3452 (1998); Phys. Rev. D 59, 074001 (1999) [Errata ibid. 60, 099906 (1999); 75, 099903 (2007)].

[3] S.R. Beane et al., Phys. Rev. D 74, 114503 (2006); J. Nagata, S. Muroya, A. Nakamura, Phys. Rev. C 80, 045203 (2009) [Erratum ibid. 84, 019904 (2011)]; Z. Fu, Phys. Rev. D 85, 074501 (2012); K. Sasaki et al. [PACS-CS Collaboration], Phys. Rev. D 89, 054502 (2014).

[4] C.B. Lang, L. Leskovec, D. Mohler, S. Prelovsek, Phys. Rev. D 86, 054508 (2012); 88, 054508 (2013); Z. Fu, K. Fu, Phys. Rev. D 86, 094507 (2012); J.J. Dudek et al. [Hadron Spectrum Collaboration], Phys. Rev. Lett. 113, 182001 (2014); D.J. Wilson, J.J. Dudek, R.G. Edwards, C.E. Thomas, Phys. Rev. D 91, 054008 (2015). 
[5] P. Estabrooks et al., Nucl. Phys. B 133, 490 (1978); D. Aston et al., Nucl. Phys. B 296, 493 (1988); Y. Cho et al., Phys. Lett. B 32, 409 (1970);

A.M. Bakker et al., Nucl. Phys. B 24, 211 (1970); D. Linglin et al., Nucl. Phys. B 57, 64 (1973); B. Jongejans et al., Nucl. Phys. B 67, 381 (1973).

[6] J.R. Peláez, A. Rodas, Phys. Rev. D 93, 074025 (2016).

[7] B. Adeva et al. [DIRAC Collaboration], Phys. Lett. B 735, 288 (2014).

[8] S. Descotes-Genon, B. Moussallam, Eur. Phys. J. C 48, 553 (2006);

P. Büttiker, S. Descotes-Genon, B. Moussallam, Eur. Phys. J. C 33, 409 (2004); B. Ananthanarayan, P. Büttiker, B. Moussallam, Eur. Phys. J. C 22, 133 (2001); B. Ananthanarayan, P. Büttiker, Eur. Phys. J. C 19, 517 (2001).

[9] R. García-Martin et al., Phys. Rev. D 83, 074004 (2011); R. Kamiński, J.R. Peláez, F.J. Ynduráin, Phys. Rev. D 74, 014001 (2006) [Erratum ibid. 74, 079903 (2006)]; J.R. Peláez, F.J. Ynduráin, Phys. Rev. D 71, 074016 (2005). 